\title{
Aplicación de la gestión de stock en el almacén de materia prima para mejora de productividad en la línea de tela de punto. Empresa textil, Lima 2017.
}

\section{Application of stock management in the raw material warehouse to improve productivity in the knitted fabric line. Textile company, Lima 2017. \\ Aplicação da gestão de estoque no armazém de matérias-primas para melhorar a produtividade na linha de malha. Empresa têxtil, Lima 2017.}

\section{Claudio Mario Gutiérrez Ascón ${ }^{(1)}$; Ronald Dávila Laguna ${ }^{(1)}$; Jaime Eduardo Gutiérrez Ascón ${ }^{(2)}$} Fecha de recepción: 06 de diciembre de 2017 Fecha de aprobación: 18 de mayo 2018

\section{RESUMEN}

Introducción: El objetivo de esta investigación fue determinar la aplicación de la gestión de stock que permita mejorar la disponibilidad de materia prima para alimentar el área de producción mejorando la productividad con los mismos o menores recursos como algodón, mano de obra, maquinaria. Material y métodos: La tesis según el tipo de estudio es una investigación aplicada, ya que busca desarrollar teorías ya existentes a procedimientos modernos. Por el diseño es Cuasi experimental, no existe muestreo, los datos se eligen con una técnica no Probabilística, sino que son elegidos intencionalmente. La poblacion esta conformada por los datos de nuestros indicadores recolectados en los formatos de recoleccion de datos. Resultados: Los resultados indican que se debe ordenar 420 toneladas de algodon cada 25 dias para asegurar el costo minimo de 5644 nuevos sole; la productividad mejora de $79.51 \%$ a $93.02 \%$. Se concluye que la aplicación de la gestion de stock contribuye con $13.51 \%$ en la mejora de productividad por lo tanto el sistema mejora en $14.52 \%$ lo que significa un ahorro por sobre costos S/ 382,800 soles que pueden ser reinvertidos en otros proyectos de mejora en la empresa IDETEX. Discusion: Son necesarias tomar medidas de emergencia para prevenir la escasez de materia prima que impiden mejorar la productividad según Cabriles( 2014) en efecto, se debe evitar reducir las posibilidades de la competencia en los mercados internacionales.

Palabras claves: Cantidad de pedido, tiempo entre pedidos, Indicadores

\begin{abstract}
Introduction: The objective of this research was to determine the application of stock management to improve the availability of raw material to feed the production area by improving productivity with the same or less resources such as cotton, labor, machinery. Material and methods: The thesis according to the type of study is an applied research, since it seeks to develop existing theories to modern procedures. Because of the design is Quasi experimental, there is no sampling, the data are chosen with a non-Probabilistic technique, but are chosen intentionally. The population is made up of the data of our indicators collected in the data collection formats. Results: The results indicate that 420 tons of cotton should be ordered every 25 days to ensure the minimum cost of 5644 new sole; productivity improves from $79.51 \%$ to $93.02 \%$. It is concluded that the application of stock management contributes $13.51 \%$ in the improvement of productivity therefore the system improves by $14.52 \%$ which means a saving over costs $\mathrm{S} / 382,800$ soles that can be reinvested in other improvement projects in the company IDETEX. Discussion: It is necessary to take emergency measures to prevent shortages of raw materials that impede improving productivity according to Cabriles (2014). In effect, it is necessary to avoid reducing the possibilities of competition in international markets.
\end{abstract}

Keywords: Order quantity, time between orders, Indicators

${ }^{(1)}$ Autor corresponsal; Claudio Mario Gutiérrez Ascón; Universidad César Vallejo- Lima Norte; Facultad de Ingeniería; Email: claudiogutierrez1@ @otmail.com teléfono 969834 072, https://orcid.org/0000-0002-1683$\underline{0506}$

${ }^{(1)}$ Ronald Dávila Laguna; Universidad César Vallejo. Filial Lima Norte; Facultad de Ingeniería; https://orcid.org/0000-0001-9886-0452

(2) Jaime Eduardo Gutiérrez Ascón; Universidad César Vallejo - Chimbote; Facultad de Ingeniería; https://orcid.org/0000-0003-4065-3359 


\section{RESUMO}

Introdução: O objetivo desta pesquisa foi determinar a aplicação da gestão de estoque para melhorar a disponibilidade de matéria-prima para alimentar a área de produção, melhorando a produtividade com o mesmo ou menos recursos, como algodão, mão-de-obra e maquinário. Material e métodos: A tese de acordo com o tipo de estudo é uma pesquisa aplicada, uma vez que procura desenvolver teorias existentes para procedimentos modernos. Devido ao design é Quasi experimental, não há amostragem, os dados são escolhidos com uma técnica não probabilística, mas são escolhidos intencionalmente. A população é composta pelos dados de nossos indicadores coletados nos formatos de coleta de dados. Resultados: Os resultados indicam que devem ser encomendadas 420 toneladas de algodão a cada 25 dias para garantir o custo mínimo de 5644 novas plantas; A produtividade melhora de $79,51 \%$ para 93,02\%. Conclui-se que a aplicação da gestão de estoque contribui com $13,51 \%$ na melhoria da produtividade, portanto, o sistema melhora em $14,52 \%$, o que significa uma economia de custos S / 382,800 solas que podem ser reinvestidas em outros projetos de melhoria em a empresa IDETEX. Discussão: é necessário tomar medidas de emergência para evitar a falta de matérias-primas que impeçam a melhoria da produtividade de acordo com Cabriles (2014). Na verdade, é necessário evitar reduzir as possibilidades de concorrência nos mercados internacionais.

Palavras-chave: quantidade de pedidos, tempo entre pedidos, indicadores

\section{Introducción}

La industria textil y de la confección en el mundo constituye una importante fuente de ingresos y empleo para muchos países, en particular para países en desarrollo. Actualmente los retos de la industria textil son conseguir la sostenibilidad debido que vivimos en el tiempo del aquí y el ahora. Una rotura de stock se refiere también a una pérdida de una venta. Hoy en día las empresas a nivel mundial hacen enormes esfuerzos para satisfacer las expectativas de sus clientes en el tiempo, costo y cantidades oportunas. Desde este punto de vista, la experiencia es extensible al mundo empresarial, la rotura de stock se refiere a dejar de servir un pedido. También es lanzar una imagen negativa sobre tu compañía al exterior, perdiéndose la confianza del cliente. Para ello se requiere encontrar siempre el balance en el stock para hacer frente a los pedidos y contar con un control de stock de seguridad en caso nuestra previsión no ha sido todo lo buena que debiera. Para esto las soluciones tecnológicas y las herramientas informáticas que se tiene implantado en muchas empresas donde se presentaban problemas de control de stock.

En el Perú cuando se pensaba en una recuperación paulatina del sector textil confecciones, tras la crisis financiera internacional del 2008-2009, se presentó un panorama desfavorable debido a serios problemas económicos que atravesaba Europa, sumada una muy lenta recuperación del mercado estadounidense. Ante esta situación, las empresas textiles se estuvieron reinventando ya sea a la conquista de nuevos mercados como también el local o a través de la producción con mayor valor agregado y para segmentos de mayores ingresos ,de manera que se ha logrado con la aplicación de la metodología de gestión de inventarios, adelantos significativos en la ciencia del almacenamiento esto hace que el producto sea valioso para el cliente porque tiene dos características, utilidad y disponibilidad, la ciencia ha hecho rápidos progresos para aumentar la utilidad. Actualmente las empresas dan cada vez mayor atención a la disponibilidad de los productos, en la que el almacenamiento desempeña un papel principal.

La empresa sólida e innovadora está establecida en Lima -Perú desde el mes de agosto del año 2000 fabrica todo tipo de telas en tejido de punto y cuenta con un complejo textil completo con más de 600 trabajadores, el proceso productivo está integrado en distintas plantas de procesamiento como desmotadoras de algodón, hilandería, tejeduría, tintorería, acabados y estampados; cuenta con una amplia y variada gama de productos como gamuza, jersey, jersey full licra, jersey hidrosedal, jersey viscosa, pique, rib, frenchterry, franela y felpa . La empresa tiene la gran ventaja de ser productor nacional, lo cual la coloca en un lugar estratégico en lo referente a tiempos de entrega, así como el evitar pago de aranceles externos al país ,nos permite rapidez y valor agregado a los productos. Para todo este proceso productivo, el inicio es fundamental, el diseño del producto es el inicio para los 
procesos textiles por lo tanto la clave fundamental radica en el diseño y desarrollo del producto, el mismo que en estos tiempos de globalización y cambios significativos, toma mayor relevancia para la organización.

Por otro lado se puede confirmar según reporte oficial de la empresa publicada en su portal que las importaciones de materia prima, insumos y maquinaria en el año 2012 fue de 14228400 millones de dólares mientras que las exportaciones fueron de 11248400 millones de dólares. Esta diferencia negativa es debida a la importación de maquinaria de última generación principalmente de Italia para incrementar valor a la cantidad y calidad de la tela. En referencia a los estados financieros, la empresa mantiene una tendencia uniforme desde el año 2011 hasta el 2016 de ingresos netos por ventas; sin embargo la tendencia del rendimiento sobre las ventas son ascendentes. Los competidores más directos son Cia Universal textil S.A., Sudamericana de fibras S.A., Consorcio textil del Pacifico S.A., Fabritex Peruana, Fijesa S.A. Empresa Algodonera S.A.

La identificación del problema resulta a partir de esta realidad expuesta que se justifica la gestión de inventarios, hecho que es un aspecto fundamental de la administración exitosa, ya que se convierte en una herramienta de medición con la que la empresa cuenta para la producción y mantenimiento de la misma. Es por ello que mantener el inventario supone un alto costo, razón por la cual la empresa no debe tener una determinada cantidad de dinero detenido en existencias excesivas, pues los objetos de un buen servicio al cliente y de una producción eficiente precisan, ser satisfechos, manteniendo los inventarios a un nivel óptimo, así como al menor costo posible, sin comprometer la calidad de los bienes ofrecidos.

El diagrama de causa efecto está presentado tomando en cuenta las opiniones de expertos y colaboradores de la empresa en donde se puede explicar las causas que originan o generan defecto en la produccion de tela de punto y el resultado siguiente explica de alguna manera algunas de las causas que originan una disminución de la productividad en la producción de tela de punto en la empresa Ideas Textiles SAC, desde luego el gráfico representa al conjunto de causas las mismas que serán evaluadas en la tabla y gráfica de Pareto en donde, como es conocido, se espera que unos pocos criterios ( 20\%) resuelvan muchos causas ( $80 \%$ ). Sin embargo es preciso afirmar que los valores porcentuales acumulados en la tabla de Pareto solamente se alcanza el valor $77,61 \%$ lo que corresponde en primer lugar a:

Tasa de rotación: Viene a ser la cantidad de veces que el inventario debe ser reemplazado durante un determinado período de tiempo, generalmente un año.

Desorden y señalización de lotes: Es la falta de identificación y ubicación de los productos dentro del proceso productivo, tanto en la linea de producción como en los almacenes de los lotes de materiales y productos terminados..

Sobrealmacenamiento: Dado que la cantidad de productos o materiales en ciertas epocas del año no han tenido la salida esperada ya sea por reprocesos o fallas en la producción de telas, crea un sobrealmacenamiento, el mismo que quita espacio a otros materiales que están a la espera de ser ingresados para el proceso de producción.

2. Materiales y métodos. Por el diseño esta tesis es Cuasi experimental, en los diseños cuasi experimentales los sujetos no se asignan al azar a los grupos ni se emparejan, sino que dichos grupos ya están formados antes del experimento: son grupos intactos (la razón por la que surgen y la manera como se formaron es independiente o aparte del experimento). (Sampieri, Fernández y Baptista, 2010, p.148).

Por el fin que perseguimos la tesis es Aplicada.

Los resultados de esta investigación se obtienen luego de la aplicación de la gestión de stock en el proceso de la línea de tela de punto de la empresa Ideas Textiles SAC. (Sampieri, Fernández y Baptista, 2010, p.50)

Por el nivel de la aplicación esta tesis es Explicativa -Descriptiva, va más allá de la descripción de conceptos, fenómenos o del establecimiento de relaciones entre conceptos; es decir, están dirigidos a responder por las causas de los eventos y fenómenos físicos o sociales. Como su nombre lo indica, su 
interés se centra en explicar por qué ocurre un fenómeno y en qué condiciones se manifiesta, o porque se relacionan dos o más variables. (Sampieri, Fernández y Baptista, 2010, p.108)

Por el enfoque esta tesis es Cuantitativa. Debe ser lo más "objetiva" posible, evitando que afecten las tendencias del investigador u otras personas. Los estudios cuantitativos siguen un patrón predecible y estructurado. Se pretende generalizar los resultados encontrados en un grupo a una colectividad mayor. La meta principal es la construcción y la demostración de teorías. (Sampieri, Fernández y Baptista, 2010, p.26)

Esta tesis por su alcance es Longitudinal. La investigación se concentra en analizar los cambios a través del tiempo de un evento, una comunidad, un fenómeno, una situación o un contexto. (Sampieri, Fernández y Baptista, 2010, p.208)

La información que ayudará a la realización del presente proyecto de investigación, será obtenida por medio de:

Observación directa. Con el fin de visualizar en forma sistemática el proceso de desarrollo de la gestión de stock en la empresa. El investigador prevé la toma de lectura según el conocimiento del proceso a investigar, dispone de las herramientas o instrumentos que aseguren la lectura exacta y levanta la información de campo en tablas no agrupadas o agrupadas también llamadas tabla estadísticas

\section{Registro histórico}

Se realiza la inspección de los reportes e informes de producción que se almacenan para efectos de verificación, evaluación, análisis, cálculos. El investigador puede tomar esta información como referencia y punto de partida para tomar conocimiento de la naturaleza del problema a resolver.

Las mediciones realizadas deben ser confiables para tomar decisiones o establecer conclusiones correctas en la investigación; por tanto un instrumento es confiable en la medida que éste produce resultados consistentes y coherentes; es decir, que en la aplicación repetida de éste al mismo sujeto u objeto produce resultados iguales

Para efecto de la determinación de validez de contenido de los instrumentos de recolección de datos en esta investigación se utilizó el juicio de expertos quienes fueron tres ingenieros industriales expertos en el tema que son de la Universidad César Vallejo.

\section{Stock o existencias.}

Las existencias son bienes tangibles para la venta en el curso ordinario del negocio o para ser consumidos en la producción de bienes o servicios para su posterior comercialización. Esta comprendido también las materias primas, productos en proceso y productos terminados conocido también como mercancías para la venta, los materiales, repuestos y accesorios para ser consumidos en la producción de bienes fabricados para la venta o en la prestación de servicios; empaques y envases y los inventarios en tránsito. El control del stock es vital para el éxito sostenido de una empresa. Cuando se abastece de productos al almacén se está amarrando dólares hoy con la esperanza de futuras utilidades.

\section{Definición operacional de gestión de stock}

La Gestión de stock es la planificación, programación y control de la cantidad óptima de existencias a producir del lote económico en función a la demanda y entregado de acuerdo al período de tiempo entre pedidos para cumplir con la línea de producción y controlar aquellos ítems que signifiquen una disminución de la producción.

\section{Métodos de Pronósticos}

Salazar López (2014) sostiene que en la actualidad podemos encontrar diferentes métodos que ya están establecidos de manera estándar. Los métodos de previsión se pueden encontrar dos tipos que son los cualitativos y cuantitativos. Para categorizarlo mejor existen los métodos de previsión que vienen a ser los cualitativos y los de proyección histórica con causales que vendrían a ser los cuantitativos. 


\section{Stock de Seguridad}

Este cálculo nos permite atender las oscilaciones no previstas del consumo que requiere la planta de producción de tela de punto para poder procesar sus productos y el plazo de aprovisionamiento.

$$
S S=Z * S * \sqrt{P E}
$$

- $\quad \mathrm{SS}=$ Stock de seguridad

- $\mathrm{Z}=$ Coeficiente de seguridad, que recoge el riesgo de rotura a asumir por fluctuaciones positivas sobre la media de la demanda.

- $\mathrm{S}=$ Desviación típica de la demanda

- $\mathrm{PE}=$ Plazo de entrega.

\section{Costos asociados a la gestión de stock}

El principal reto que enfrenta la organización cuando gestiona sus inventarios es el de mantener en equilibrio la relación entre servicio prestado al cliente y los costos asociados al sistema es por eso la necesidad de una buena estimación de los mismos. (Cruelles 2012 p. 53)

\section{Lote óptimo de pedido (Economic Order Quantity-EOQ)}

Se trata de un modelo determinista, pues parte de la hipótesis de que las ventas de la empresa son conocidas y que se reparten uniformemente a lo largo del año. (Suárez, 2012, p. 89)

Para determinar el modelo vamos a utilizar las siguientes variables:

P: Precio de adquisición de cada unidad de producto

V: Cantidad de producto vendido al año.

E: Coste de preparación del pedido. Recoge los costes de administración relacionados con la obtención del pedido.

$$
\begin{aligned}
& \mathrm{Q}=\sqrt[2]{2 K D / g} \\
& \mathrm{Q}=\text { Volumen óptimo de pedido } \\
& \mathrm{K}=\text { Coste de realızacıon de un pedıdo. } \\
& \mathrm{D}=\text { Volumen de demanda } \\
& \mathrm{g}=\text { Coste anual de mantener } \\
& \text { una unıaad de proaucto. }
\end{aligned}
$$

\section{Tiempo entre pedidos}

Una vez calculado el pedido óptimo, se puede calcular el número de pedidos $\mathrm{N}$ y así, es posible calcular el tiempo T entre pedidos cuando se considera el año de 360 días; por otro lado, el número de pedidos anuales se calculando la demanda por volumen de pedidos en la gestión de las existencias (Suárez p.94).A continuación se expresa con la siguiente formula:

$$
\mathrm{T}=\frac{360 \text { dias }}{\mathrm{N}}
$$

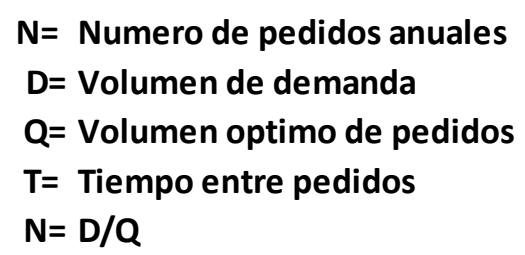

$$
\mathbf{N}=\mathbf{D} / \mathbf{Q}
$$


El índice de productividad expresa el buen aprovechamiento de todos y cada uno de los factores dela producción, los criterios e importantes en un período definido. (García, 2011, p. 17).

El uso de un solo recurso de entrada para medir la productividad, se conoce como productividad de un solo factor. Sin embargo, un panorama más amplio de la productividad es la productividad de múltiples factores, la cual incluye todos los insumos o entradas (por ejemplo, capital, mano de obra, material, energía). La productividad de múltiples factores también se conoce como productividad de factor total. La productividad de múltiples factores se calcula combinando las unidades de entrada como se muestra a continuación: (Heizer y Render, 2009 p.15)

$$
\text { Productividad }=\frac{\text { Salida }}{\text { Mano de obra }+ \text { material }+ \text { energía }+ \text { capital }+ \text { otros }}
$$

\section{Hipótesis General.}

La aplicación de la gestión de stock en el almacén de materia prima mejora la productividad en la línea de tela de punto de la empresa Ideas Textiles S.A.C.

\section{H0. Hipótesis nula.}

La aplicación de la gestión de stock en el almacén de materia prima no mejora la productividad en la línea de tela de punto de la empresa Ideas Textiles SAC.

\section{Objetivo general.}

Determinar cómo la aplicación de la gestión de stock en el almacén de materia prima mejora la productividad en la línea de tela de punto de la empresa Ideas Textiles S.A.C.

\section{Diseño de Investigación}

Por el fin que perseguimos la tesis es Aplicada.

Los resultados de esta investigación se obtienen luego de la aplicación de la gestión de stock en el proceso de la línea de tela de punto de la empresa Ideas Textiles SAC. (Sampieri, Fernández y Baptista, 2010, p.50).

\section{Investigación Descriptiva- Explicativa}

Por el nivel de la aplicación esta tesis es Explicativa -Descriptiva, va más allá de la descripción de conceptos, fenómenos o del establecimiento de relaciones entre conceptos; es decir, están dirigidos a responder por las causas de los eventos y fenómenos físicos o sociales. Como su nombre lo indica, su interés se centra en explicar por qué ocurre un fenómeno y en qué condiciones se manifiesta, o porque se relacionan dos o más variables. (Sampieri, Fernández y Baptista, 2010, p.108)

\section{Investigación Cuantitativa}

Por el enfoque esta tesis es Cuantitativa. Debe ser lo más "objetiva" posible, evitando que afecten las tendencias del investigador $u$ otras personas. Los estudios cuantitativos siguen un patrón predecible y estructurado. Se pretende generalizar los resultados encontrados en un grupo a una colectividad mayor. La meta principal es la construcción y la demostración de teorías. (Sampieri, Fernández y Baptista, 2010, p.26)

\section{Resultados}

\section{Análisis Pre test}

Brecha entre existencias de almacén de algodón y producción.

En la tabla se observa que debido a la improvisación de las compras de algodón según los meses de agosto a noviembre, no se registran ingresos de algodón ya que el almacen venía de una experiencia 
de sobrealmacenamiento de meses anteriores. Este hecho generó un déficit o rotura de stock para diciembre 2015 con 109 toneladas y enero 2016 con 308 toneladas de diferencia entre entre el requerimiento de producción y las existencias de algodón en el almacén situación que obligó a compras de emergencia de proveedores nacionales y extranjeros. Situación similar se presentó en noviembre y diciembre 2016 y en la figura 1se observa la brecha que se inicia en noviembre hasta febrero $2016 \mathrm{y}$ se repite al fin de este año

Tabla 1: Brecha entre existencias y producción 2015 - 2016.

\begin{tabular}{|c|c|c|c|c|c|}
\hline & Año & $\begin{array}{l}\text { INGRESO } \\
\text { MATERIA } \\
\text { PRIMA } \\
\text { ( Algodón -Kg) }\end{array}$ & $\begin{array}{c}\text { EXISTENCIAS } \\
\text { ALMACEN } \\
\text { MATERIA PRIMA } \\
\text { (kg) }\end{array}$ & PRODUCCIÓN (kg) & $\begin{array}{c}\text { BRECHA: } \\
\text { EXITENCIAS - } \\
\text { PRODUCCIÓN } \\
(\mathbf{k g})\end{array}$ \\
\hline \multicolumn{5}{|c|}{ saldo del año anterior) stock inicial------------ } & \\
\hline 1 & Ene-15 & 303526 & 745012 & 258514 & 486498 \\
\hline 2 & Feb-15 & 701906 & 1166138 & 280780 & 885359 \\
\hline 3 & Mar-15 & 295445 & 1215751 & 245833 & 969918 \\
\hline 4 & Abr-15 & 445810 & 1361148 & 300412 & 1060736 \\
\hline 5 & May-15 & 450924 & 1512869 & 299203 & 1213666 \\
\hline 6 & Jun-15 & 329080 & 1561013 & 280936 & 1280078 \\
\hline 7 & Jul-15 & 51253 & 1309933 & 302334 & 1007599 \\
\hline 8 & Ago-15 & 0 & 1004804 & 305128 & 699676 \\
\hline 9 & Set-15 & 0 & 756179 & 248626 & 507553 \\
\hline 0 & Oct-15 & 0 & 570955 & 185224 & 385732 \\
\hline 1 & Nov-15 & 0 & 320535 & 250420 & 70115 \\
\hline 2 & Dic-15 & 175155 & 193166 & 302524 & -109358 \\
\hline 3 & Ene-16 & 117590 & 1079 & 309677 & -308599 \\
\hline 4 & Feb-16 & 729772 & 385173 & 345678 & 39495 \\
\hline 5 & Mar-16 & 435670 & 470563 & 350279 & 120284 \\
\hline 6 & Abr-16 & 575500 & 750410 & 295653 & 454757 \\
\hline 7 & May-16 & 733116 & 998221 & 485306 & 512915 \\
\hline 8 & Jun-16 & 502451 & 1079283 & 421389 & 657894 \\
\hline 9 & Jul-16 & 628502 & 1334894 & 372891 & 962002 \\
\hline 0 & Ago-16 & 336810 & 1172073 & 499630 & 672443 \\
\hline 1 & Set-16 & 0 & 656199 & 515874 & 140325 \\
\hline 2 & Oct-16 & 158540 & 421880 & 392859 & 29021 \\
\hline 3 & Nov-16 & 0 & 32882 & 388998 & -356116 \\
\hline 4 & Dic-16 & 0 & -295321 & 328203 & -623523 \\
\hline & & & -295321 & 7966370 & \\
\hline
\end{tabular}

Existencias de ALGODON con inventario inicial y PRODUCCIÓN 2015 - 2016

2000000

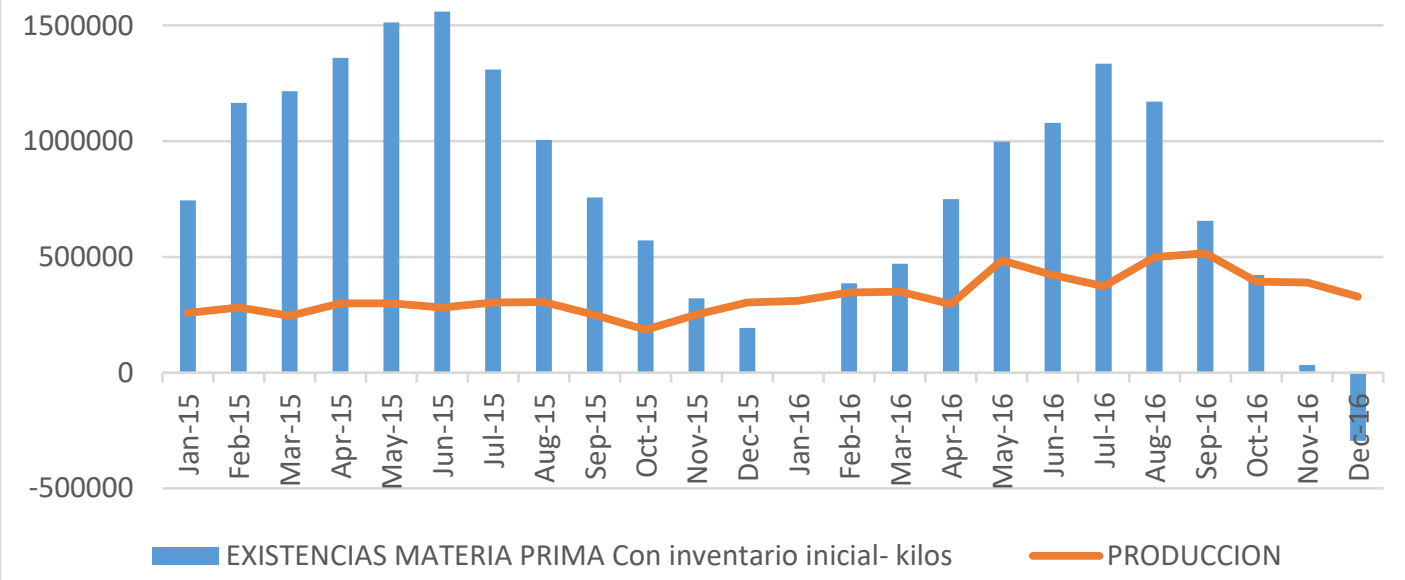

Figura1: Brecha entre existencias almacén materia prima y producción 2015 - 2016. 
Tabla 2: Pronóstico de la demanda de algodón año 2017

PONOSTICO DE

DEMANDA 2017

\begin{tabular}{|ll|}
\hline enero & 451579 \\
\hline febrero & 500731 \\
marzo & $\mathbf{4 8 9 9 0 2}$ \\
abril & $\mathbf{3 9 9 8 2 7}$ \\
mayo & $\mathbf{6 4 6 7 9 7}$ \\
junio & 563448 \\
\hline julio & 594525 \\
agosto & 596601 \\
septiembre & 482765 \\
octubre & 364102 \\
noviembre & 486522 \\
diciembre & 573079 \\
\hline
\end{tabular}

En la tabla del pronóstico para el año 2017, se observa que los valores pronosticados del período de febrero a junio siguen la misma tendencia ascendente que los valores de la demanda 2015 a 2016. Esta información, servirá para determinar el modelo EOQ del inventario de algodón en el post test de esta investigación.

\section{Nivel de productividad pre test}

Se determina la productividad promedio total para los 20 períodos es de 79,51\% situación que se espera mejorar con la gestión de stock de algodón

Tabla 3. Nivel de productividad Pre Test.

\begin{tabular}{|c|c|c|c|c|c|c|c|}
\hline \multirow{2}{*}{\begin{tabular}{|r} 
Semana \\
Sem.34
\end{tabular}} & \multicolumn{3}{|c|}{ Fecha: } & EFICIENCIA & EFICACIA & $\begin{array}{r}\text { PRODUCTIVIDAD } \\
(\%)\end{array}$ & PRE TEST \\
\hline & 15/08/2016 & a & $21 / 08 / 2016$ & $90.27 \%$ & $99.83 \%$ & $90.12 \%$ & \\
\hline Sem.35 & 22/08/2016 & a & 28/08/2016 & $91.05 \%$ & $99.83 \%$ & $90.90 \%$ & \\
\hline Sem.36 & 29/08/2016 & a & 4/09/2016 & $90.21 \%$ & $99.83 \%$ & $90.06 \%$ & \\
\hline Sem.37 & 5/09/2016 & a & $11 / 09 / 2016$ & $87.27 \%$ & $98.66 \%$ & $86.11 \%$ & \\
\hline Sem.38 & 12/09/2016 & a & 18/09/2016 & $82.64 \%$ & $98.66 \%$ & $81.54 \%$ & \\
\hline Sem.39 & 19/09/2016 & a & $25 / 09 / 2016$ & $86.39 \%$ & $98.66 \%$ & $85.24 \%$ & \\
\hline Sem.40 & 26/09/2016 & a & 2/10/2016 & $85.53 \%$ & $98.66 \%$ & $84.39 \%$ & \\
\hline Sem.41 & 3/10/2016 & $a$ & 9/10/2016 & $88.72 \%$ & $98.57 \%$ & $87.45 \%$ & \\
\hline Sem.42 & $10 / 10 / 2016$ & a & $16 / 10 / 2016$ & $81.49 \%$ & $98.57 \%$ & $80.32 \%$ & \\
\hline Sem.43 & $17 / 10 / 2016$ & a & 23/10/2016 & $83.03 \%$ & $98.57 \%$ & $81.84 \%$ & \\
\hline Sem.44 & 24/10/2016 & a & $30 / 10 / 2016$ & $84.02 \%$ & $98.57 \%$ & $82.81 \%$ & \\
\hline Sem.45 & $31 / 10 / 2016$ & a & 6/11/2016 & $83.33 \%$ & $98.57 \%$ & $82.14 \%$ & \\
\hline Sem.46 & 7/11/2016 & a & 13/11/2016 & $59.24 \%$ & $86.41 \%$ & $51.19 \%$ & \\
\hline Sem.47 & $14 / 11 / 2016$ & a & 20/11/2016 & $79.33 \%$ & $84.47 \%$ & $67.01 \%$ & \\
\hline Sem.48 & $21 / 11 / 2016$ & a & $27 / 11 / 2016$ & $85.00 \%$ & $84.47 \%$ & $71.80 \%$ & \\
\hline Sem.49 & $28 / 11 / 2016$ & a & 4/12/2016 & $84.64 \%$ & $84.47 \%$ & $71.49 \%$ & \\
\hline Sem.50 & $5 / 12 / 2016$ & a & $11 / 12 / 2016$ & $83.15 \%$ & $94.47 \%$ & $78.55 \%$ & \\
\hline Sem.51 & $12 / 12 / 2016$ & a & 18/12/2016 & $75.68 \%$ & $94.47 \%$ & $71.49 \%$ & \\
\hline Sem.52 & $19 / 12 / 2016$ & a & $25 / 12 / 2016$ & $86.01 \%$ & $94.47 \%$ & $81.25 \%$ & \\
\hline Sem.53 & $26 / 12 / 2016$ & $\mathrm{a}$ & $31 / 12 / 2016$ & $78.86 \%$ & $94.47 \%$ & $74.50 \%$ & \\
\hline & & & PRODUC & IDAD PRO & IO TOTAL & $79.51 \%$ & \\
\hline
\end{tabular}

\section{Implementación de la gestión de stock}

La gestión de stock está determinada por las dos dimensiones identificadas en la Operacionalización de variables como son la cantidad o lote óptimo de pedido y el tiempo entre pedidos; para este 
propósito se empleó la demanda pronosticada de algodón según tabla donde la información pronosticada sirvió para el cálculo de la cantidad o lote económico.

- Cantidad óptima a ordenar de materia prima:

Según la tabla 4 se observa que los datos requeridos por el modelo para determinar el lote económico se requiere de información obtenida en cuadros precedentes como el pronóstico de la demanda, costo de almacenamiento, costo de lanzar el pedido.

\begin{tabular}{|c|c|c|}
\hline \multirow{11}{*}{$\begin{array}{l}\text { Tabla 4: } \\
\text { de entrada } \\
\text { Análisis }\end{array}$} & Descripción & $\begin{array}{c}\text { Entrada Año } \\
2017\end{array}$ \\
\hline & Demanda & 6150 \\
\hline & Costo de ordenar & 192.71 \\
\hline & Costo de almacenar una unidad por año & 13.44 \\
\hline & Costo unitario de escasez por año & M \\
\hline & Costo unitario de escasez independiente del tiempo & - \\
\hline & $\begin{array}{l}\text { Proporción de aprovisionamiento o producción por } \\
\text { año }\end{array}$ & M \\
\hline & Tiempo de espera para un nuevo orden en el año & - \\
\hline & Costo de adquisición de una unidad sin descuento & 6250 \\
\hline & Número de roturas de descuento (cantidades) & - \\
\hline & Cantidad de la orden si es conocida & - \\
\hline
\end{tabular}

Cuantitativo con WINQSB del modelo 8: Teoría y gestión de inventarios.

\begin{tabular}{|c|c|c|c|c|}
\hline \multicolumn{5}{|c|}{ st Analysis per year for Gestion de stock año 2017 (Toneladas) } \\
\hline 05-31-2017 & Input Data & Value & Economic Order Analysis & Value \\
\hline 1 & Demand per year & 6150 & Order quantity & 419.9572 \\
\hline 2 & Order (setup) cost & $\$ 192.7100$ & Maximum inventory & 419.9572 \\
\hline 3 & Unit holding cost per year & $\$ 13.4400$ & Maximum backorder & 0 \\
\hline 4 & Unit shortage cost & & Order interval in year & 0.0683 \\
\hline 5 & per year & $M$ & Reorder point & 0 \\
\hline 6 & Unit shortage cost & & & \\
\hline 7 & independent of time & 0 & Total setup or ordering cost & $\$ 2822.1120$ \\
\hline 8 & Replenishment/production & & Total holding cost & $\$ 2822.1130$ \\
\hline 9 & rate per year & $M$ & Total shortage cost & 0 \\
\hline 10 & Lead time in year & 0 & Subtotal of above & $\$ 5644.2250$ \\
\hline 11 & Unit acquisition cost & $\$ 6250.0000$ & & \\
\hline 12 & & & Total material cost & $\$ 38437500.0000$ \\
\hline \multicolumn{5}{|l|}{13} \\
\hline 14 & & & Grand total cost & $\$ 38443140.0000$ \\
\hline
\end{tabular}

Figura 2 : Reporte de salida modelo EOQ

Donde se reportan los resultados como:

1. Lote económico de pedido $=419$ toneladas.

2. Tiempo entre pedidos $=0,0683 \times 365=24,92$ días ( 25 días) 

3. Costo total de ordenar unidades
$=2822,11$ soles
4. Costo total de mantener unidades
$=2822,11$ soles
5. Costo total de compra de material
$=38437500$ soles
6. Costo total
$=38443140$ soles

En la figura 3 se observa el costo mínimo de mantener el inventario y el lote óptimo de pedido de 420 toneladas que se piden cada 25 días para atender la producción de tela de punto cuando se intersectan los costó de hacer el pedido y mantener el inventario en el almacén.

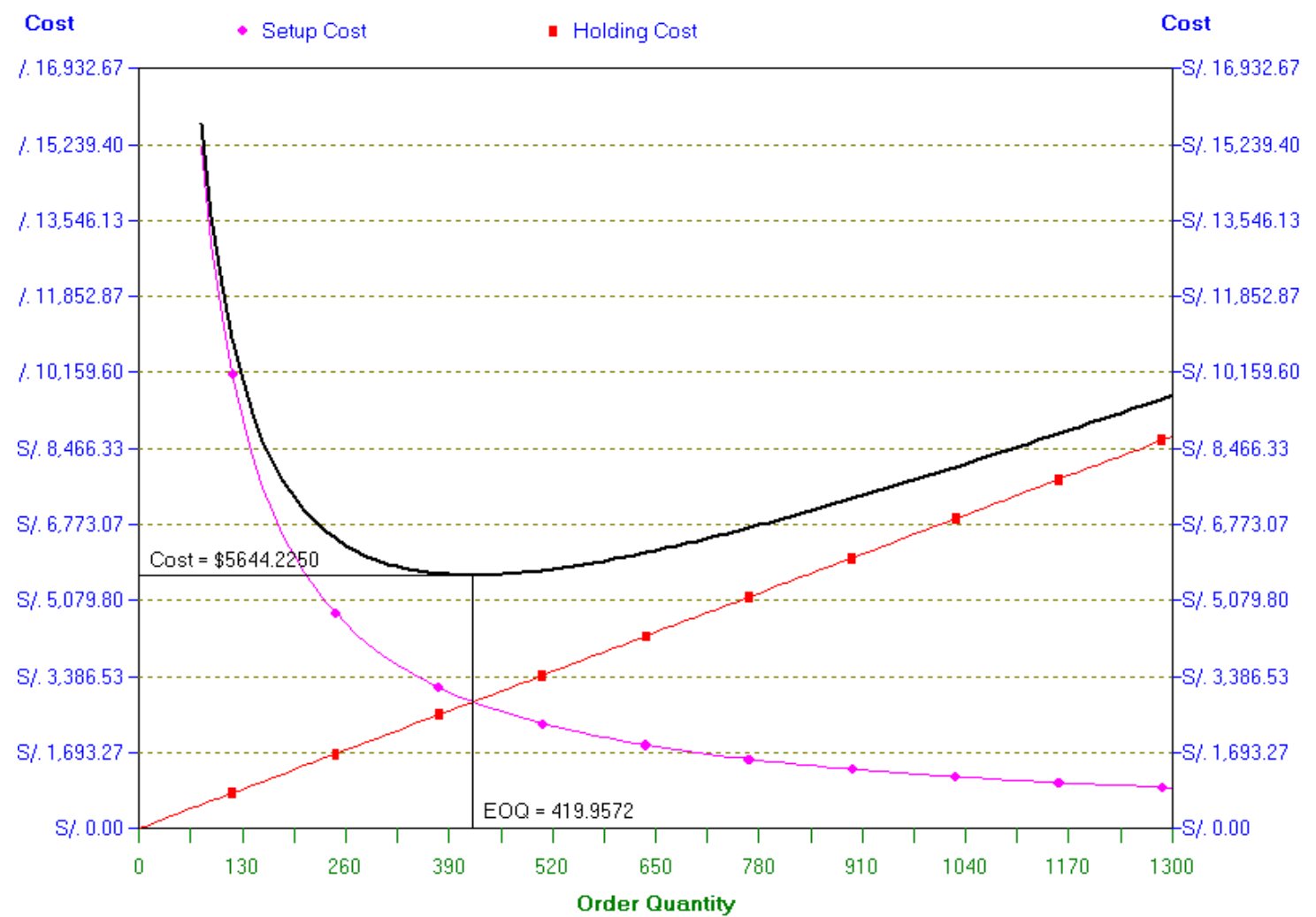

Figura 3 EOQ: Cantidad Óptima, Costo Total mínimo.

\section{Eficiencia de productividad post test}

Según la tabla 5 la vista de los resultados alcanzados, se encontró que para completar el proceso de la gestión del inventario después de planificado y según tabla, se programó las fechas en las que las cantidades de lote óptimo debe ser entregado el algodón; la gestión del inventario de algodón culmina con el control de los niveles de stock, donde se evidencia que no existen límites peligrosos de desabastecimiento o roturas que pongan en riesgo la producción y obligue a los responsables de compras a pagar sobrecostos por compras de emergencia para cumplir con el requerimiento de producción. El promedio de eficiencia es $95,36 \%$.

Tabla 5 : Eficiencia de productividad Post test: Febrero - junio 2017. 


\begin{tabular}{|c|c|c|c|c|c|c|c|c|}
\hline & & & & & & & GESTIÓN & DE STOCK \\
\hline & & & & & & & CONTROL EOQ & PROGRAMACIÓN \\
\hline & Coman & Fonho & DEMANDA & \begin{tabular}{c|} 
CANTIDAD DE \\
INSUMOS
\end{tabular} & $\begin{array}{l}\text { CANTIDAD DE } \\
\text { INSUMOS }\end{array}$ & EFICIENCIA= & EXISTENCIAS DE & EOQ \\
\hline & Semana & recina: & PRONOSTICADA & $\begin{array}{c}\text { PROGRAMADOS (IP) } \\
(\mathrm{t})\end{array}$ & $\begin{array}{l}\text { UTILIZADOS } \\
\text { (IU) } \quad \text { (t) }\end{array}$ & IP /IU & 2017(toneladas) & 25-Ene \\
\hline 1 & Sem.6 & $06-02-17$ a 12-02-17 & Feb-17 & 166.67 & 175.1 & $95.19 \%$ & 344.85 & 519.95 \\
\hline 2 & Sem.7 & $13-02-17$ a $19-02-17$ & 50001 & 166.67 & 173.5 & $96.06 \%$ & 171.35 & $19-\mathrm{Feb}$ \\
\hline 3 & Sem. 8 & $20-02-17$ a $26-02-17$ & 500.01 & 166.67 & 174.8 & $95.35 \%$ & 416.5 & \\
\hline 4 & Sem.9 & $27-02-17$ a $05-03-17$ & Mar-17 & 97.98 & 102.2 & $95.87 \%$ & 314.3 & 419.95 \\
\hline 5 & Sem. 10 & 06-03-17 a 12-03-17 & & 97.98 & 101.7 & $96.34 \%$ & 212.6 & \\
\hline 6 & Sem. 11 & $13-03-17$ a $19-03-17$ & 1899 & 97.98 & 102.5 & $95.59 \%$ & 110.1 & 16-Mar \\
\hline 7 & Sem.12 & 20-03-17 a 26-03-17 & 489.9 & 97.98 & 102.9 & $95.22 \%$ & 427.15 & \\
\hline 8 & Sem.13 & $27-03-17$ a $02-04-17$ & & 97.98 & 101.8 & $96.25 \%$ & 325.35 & 419.95 \\
\hline 9 & Sem. 14 & 03-04-17 a 09-04-17 & Abr-17 & 99.96 & 104.5 & $95.66 \%$ & 220.85 & \\
\hline 10 & Sem.15 & $10-04-17$ a $16-04-17$ & & 99.96 & 105.6 & $94.66 \%$ & 535.2 & $10-A b r$ \\
\hline 11 & Sem.16 & $17-04-17$ a $23-04-17$ & 399.84 & 99.96 & 104.9 & $95.29 \%$ & 430.3 & 419.95 \\
\hline 12 & Sem.17 & 24-04-17 a 30-04-17 & & 99.96 & 103.4 & $96.67 \%$ & 326.9 & \\
\hline 13 & Sem.18 & $01-05-17$ a $07-05-17$ & May-17 & 161.69 & 169.1 & $95.62 \%$ & 157.8 & 5-May \\
\hline 14 & Sem.19 & $08-05-17$ a $14-05-17$ & & 161.69 & 170.6 & $94.78 \%$ & 407.15 & \\
\hline 15 & Sem.20 & $15-05-17$ a $21-05-17$ & 646.76 & 161.69 & 171.5 & $94.28 \%$ & 235.65 & 419.95 \\
\hline 16 & Sem.21 & $22-05-17$ a $28-05-17$ & & 161.69 & 173.5 & $93.19 \%$ & 62.15 & \\
\hline 17 & Sem.22 & $29-05-17$ a $04-06-17$ & Jun-17 & 140.86 & 147.4 & $95.56 \%$ & 334.7 & 30-May \\
\hline 18 & Sem.23 & 05-06-17 a 11-06-17 & & 140.86 & 149.5 & $94.22 \%$ & 185.2 & \\
\hline 19 & Sem.24 & $12-06-17$ a $18-06-17$ & 563.44 & 140.86 & 146.4 & $96.22 \%$ & 38.8 & \\
\hline 20 & Sem.25 & $19-06-17$ a $25-06-17$ & & 140.86 & 147.9 & $95.24 \%$ & 310.85 & 24-Jun \\
\hline & & & & EFICIENCIA & OMEDIO= & $95.36 \%$ & & 419.95 \\
\hline
\end{tabular}

Tabla 6 : Eficacia de productividad Post Test: Febrero - junio 2017.

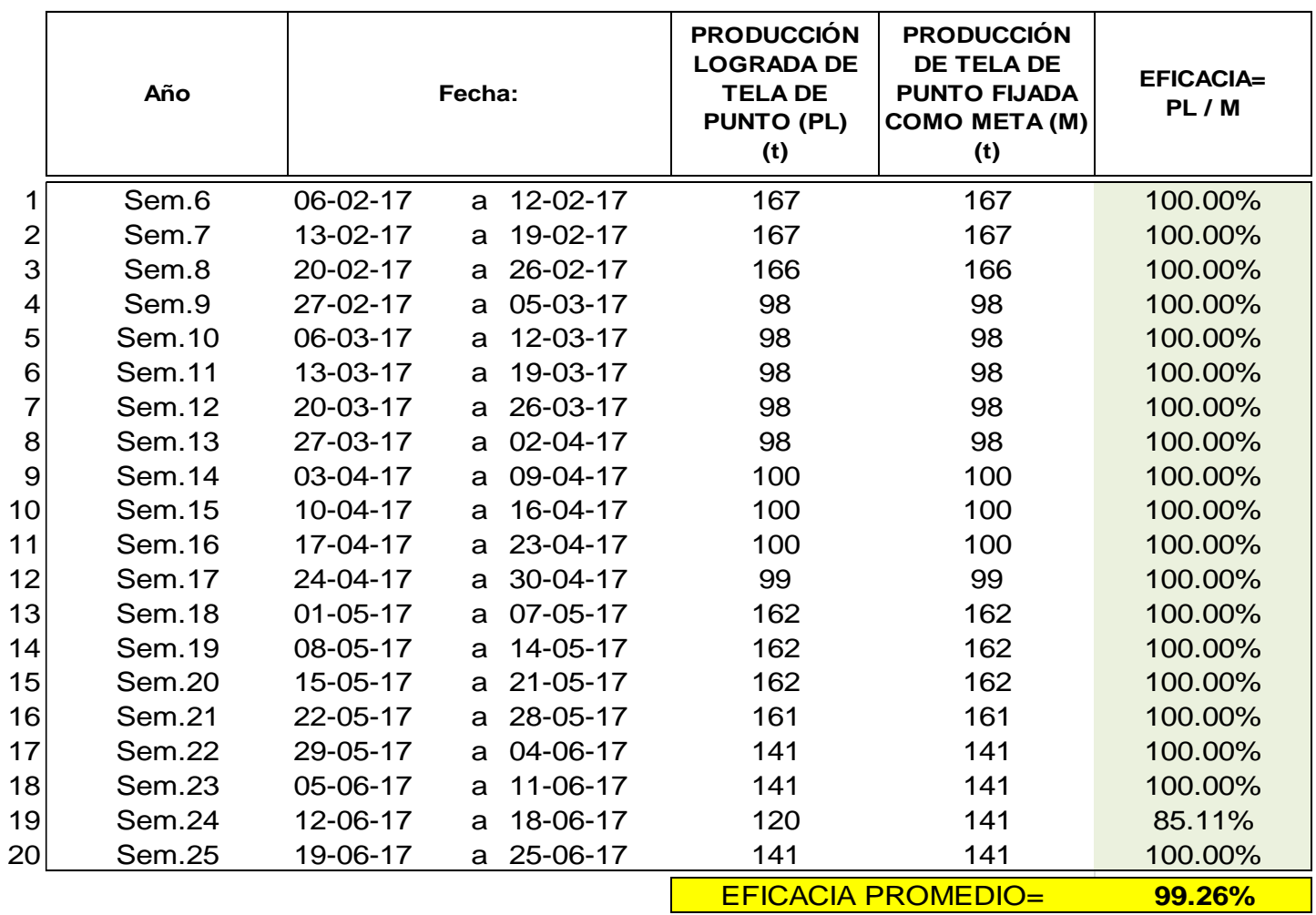

Tabla 1: Productividad post test: Febrero - junio 2017. 


\begin{tabular}{|c|c|c|c|c|c|}
\hline Semana & & cha: & \begin{tabular}{|c} 
EFICIENCIA \\
POST TEST (A) \\
(\%)
\end{tabular} & $\begin{array}{c}\text { EFICACIA } \\
\text { POST TEST (B) } \\
(\%)\end{array}$ & $\begin{array}{l}\text { PRODUCTIVIDAD } \\
\text { POST TEST = } \\
\text { A x B (\%) }\end{array}$ \\
\hline Sem. 6 & $06-02-17$ & a $12-02-17$ & $95.19 \%$ & $100.00 \%$ & $95.19 \%$ \\
\hline Sem. 7 & $13-02-17$ & a $19-02-17$ & $96.06 \%$ & $100.00 \%$ & $96.06 \%$ \\
\hline Sem. 8 & $20-02-17$ & a $26-02-17$ & $95.35 \%$ & $100.00 \%$ & $95.35 \%$ \\
\hline Sem. 9 & $27-02-17$ & a $05-03-17$ & $95.87 \%$ & $100.00 \%$ & $95.87 \%$ \\
\hline Sem. 10 & $06-03-17$ & a $12-03-17$ & $96.34 \%$ & $100.00 \%$ & $96.34 \%$ \\
\hline Sem. 11 & $13-03-17$ & a $19-03-17$ & $95.59 \%$ & $100.00 \%$ & $95.59 \%$ \\
\hline Sem. 12 & $20-03-17$ & a $26-03-17$ & $95.22 \%$ & $100.00 \%$ & $95.22 \%$ \\
\hline Sem. 13 & 27-03-17 & a $02-04-17$ & $96.25 \%$ & $100.00 \%$ & $96.25 \%$ \\
\hline Sem. 14 & $03-04-17$ & a $09-04-17$ & $95.66 \%$ & $100.00 \%$ & $95.66 \%$ \\
\hline Sem. 15 & $10-04-17$ & a $16-04-17$ & $94.66 \%$ & $100.00 \%$ & $94.66 \%$ \\
\hline Sem. 16 & $17-04-17$ & a $23-04-17$ & $95.29 \%$ & $100.00 \%$ & $95.29 \%$ \\
\hline Sem. 17 & $24-04-17$ & a $30-04-17$ & $96.67 \%$ & $100.00 \%$ & $96.67 \%$ \\
\hline Sem. 18 & $01-05-17$ & a $07-05-17$ & $95.62 \%$ & $100.00 \%$ & $95.62 \%$ \\
\hline Sem. 19 & $08-05-17$ & a $14-05-17$ & $94.78 \%$ & $100.00 \%$ & $94.78 \%$ \\
\hline Sem. 20 & $15-05-17$ & a $21-05-17$ & $94.28 \%$ & $100.00 \%$ & $94.28 \%$ \\
\hline Sem. 21 & $22-05-17$ & a $28-05-17$ & $93.19 \%$ & $100.00 \%$ & $93.19 \%$ \\
\hline Sem. 22 & 29-05-17 & a $04-06-17$ & $95.56 \%$ & $100.00 \%$ & $95.56 \%$ \\
\hline Sem. 23 & $05-06-17$ & a $11-06-17$ & $94.22 \%$ & $100.00 \%$ & $94.22 \%$ \\
\hline Sem. 24 & $12-06-17$ & a $18-06-17$ & $96.22 \%$ & $85.11 \%$ & $81.89 \%$ \\
\hline Sem. 25 & $19-06-17$ & a $25-06-17$ & $95.24 \%$ & $100.00 \%$ & $95.24 \%$ \\
\hline \multicolumn{5}{|c|}{ PRODUCTIVIDAD PROMEDIO= } & $94.65 \%$ \\
\hline
\end{tabular}

\section{Mejora de la productividad}

Según la tabla 8 la productividad se muestra en forma de crecimiento teniendo una mejora resaltante de febrero a junio con una diferencia de $15,14 \%$ esta diferencia es reflejada por la mejora en la eficiencia y eficacia de la empresa textil.

Tabla 8: Resumen de productividad pretest - post test

\begin{tabular}{r|ccc|}
\cline { 2 - 4 } & EFICIENCIA & EFICACIA & PRODUCTIVIDAD \\
\cline { 2 - 4 } Pre test: & $\mathbf{8 3 . 2 9 \%}$ & $95.23 \%$ & $79.51 \%$ \\
Post test: & $95.36 \%$ & $99.26 \%$ & $94.65 \%$ \\
\cline { 2 - 4 } Contribución $=$ & $\mathbf{1 2 . 0 7 \%}$ & $\mathbf{4 . 0 3} \%$ & $\mathbf{1 5 . 1 4 \%}$ \\
\cline { 2 - 4 }
\end{tabular}

\section{Discusión}

Como resultado de la investigación según la tabla 8 se observa que la productividad en la empresa está establecida mediante los indicadores de eficiencia y eficacia cuyo producto da como resultado una mejora en la productividad de $79,51 \%$ a $94,65 \%$

Concuerdo con Abarca A. Antonio. (2010) con su tesis: Sistema de agentes para control de stock de almacén basado en identificación por radiofrecuencia. (Ingeniero Industrial). La Mancha, España: Universidad de Castilla La Mancha, Departamento de Ingeniería Eléctrica, Electrónica y automática. 
Cuyo objetivo fue encontrar un equilibrio entre dos fuerzas que van en sentido opuesto y que contribuyen a determinar el volumen de stock almacenamiento en la empresa: Por un lado, no es deseable entrar en rotura de stock, provocando la paralización de las ventas, con todas las consecuencias que ello acarrea como la imagen de la empresa, devolución de pedidos, pérdidas económicas. El tipo de investigación fue Aplicada, diseño de investigación Cuantitativo, exploratorio y descriptivo, la población tomada dentro de la empresa y la muestra tomada en área de almacén; el instrumento usado fue entrevistas estructuradas y medición de campo, las entrevista informales y con carácter de diagnóstico. Es así que llega a la conclusión que con la ubicación adecuada de las antenas RFID se tiene una localización por zonas de todos los productos del stock, facilitando de esta forma el acceso a los productos y optimizando la ruta de los operarios a la hora de complementar los pedidos. También se reducen significativamente las pérdidas desconocidas productos que desaparecen del almacén sin saber el motivo o que simplemente aparecen en el sistema y físicamente no están en las instalaciones. Ante estos resultados, se puede decir que el investigador buscaba resolver el equilibrio entre las dos fuerzas el sobre almacenamiento y la rotura del stock en donde ambas tienen efecto negativo en los resultados del balance económico de la empresa.

Se concluye de los resultados obtenidos en el trabajo de investigación que la aplicación de la gestión de stock en el almacén de materia prima, mejora en 19,04 \% la productividad pre test de 79,51\% a $94,85 \%$ resultados que son confirmados por la prueba estadística de diferencia de hipótesis, contrastando al 5\% de significancia o 95\% de confianza que la productividad post test es mayor que la productividad pre test.

Se recomienda implementar un sistema de gestión de stock en el almacén de materia prima, para mejorar la productividad aplicando indicadores cuantitativos Kpi y medir todo lo que sebe mejorar la problemática como el metodo EOQ el cual nos proporciona las cantidades adecuadas en los tiempos apropiados y evitar que se genere sobrealmacenamiento de materiales ni rotura de stock.

\section{Referencias}

Cabriles G., Isabel. ( 2012) Propuesta de un sistema de control de inventario de stock de seguridad para mejorar la gestión de compras de materia prima, repuestos e insumos de la empresa Balgres C.A. (Administración del Transporte). Caracas, Venezuela.

Cruelles, José. Productividad e incentivos: cómo hacer que los tiempos de fabricación se cumplan. 1a ed. México. Alfaomega Grupo Editor, S.A. 2013. 220pp. ISBN: 9786077075783.

Garcia, Alfonso. Productividad y reducción de costos. Para la pequeña y mediana industria 2a ed. México. Trillas. 2011.304 pp. ISBN: 9786071707338.

Heiser, Jay y RENDER. Principios de administración de operaciones. 7a ed. México. Pearson Educación. 2009. 752pp. ISBN: 9786074420999.

Suárez, María. Gestión de Inventarios. Una nueva fórmula de calcular la competitividad. $1^{\text {a }}$ ed. Bogotá. Ediciones de la U. 2012. 182pp. ISBN: 9789587620610.

Sampieri, Roberto, Fernandez, Carlos y Baptista María, Metodología de la Investigación. $5^{\text {a }}$ ed. México: McGraw-Hill, 2010. 656p. ISBN: 9786071502919

Tejero Jorge (2013). En su estudio, “Aplicación de productividad a una empresa de servicios”, para otorgarle el título de ingeniero industrial en la universidad de Piura, Perú. 